\title{
THE TEACHING OF ELEMENTARY PSYCHOLOGY IN COLLEGES AND UNIVERSITIES WITH LAB- ORATORIES.
}

\author{
By E. C. Sanford, \\ Clark Universily.
}

To obtain data on the teaching of first year classes in psychology in such institutions, the following questionary was sent to instructors in psychology in the leading institutions east and west with the request that they would furnish the information desired, making replies to the questions, if so minded, or adopting some other form of presentation, if they should prefer.

\section{First Year Psychology}

\section{INFORMATION IS DESIRED ON THR FOLLOWING POINTS}

\section{A. Purpose of the course as now given:}

Is it looked upon as a means to liberal culture chiefly, as an introduction to philosophical studies in general, as a subject having useful applications, as a science to be cultivated for itself?

If all of these ideals enter, or several of them, please indicate their relative importance.

\section{B. Academic status of the course:}

Is it a free elective, a required part of cel tain groups, a required preliminary for courses other than advanced psychology, an optional course?

Does your introductory course as now given run thiough an entire year? How many periods a week? How much laboratory, how mucb class work?

How many usually register for the course? Are they handled in one or several sections? About what proportion go on to take further work in psychology?

\section{Content of the course:}

On what is the chief emphasis laid in the course: psychological theory, physiological and experimental matters, relations to daily life, pedagogicai and other applications? 
Do you try to do anything with comparative psychology (animals), child psychology, mental and nervous diseases, psychological questions of the day such as hypnotism, telepathy, mental healing, psychology of testimony and the like?

If all or several of these topics are treated, please give the relative importance attached to them.

\section{Methods of instruction:}

Lectures, text-book (one chiefly, or several at a time), collateral reading, reports by students, themes, discussions, seminary work, individual conferences-which of these, or which combination of them, do you find most satisfactory?

Do you give the students special pedagogical assistance in the way of outlines, reviews with or without special review questions, quizzes, or examinations other than those for the determination of academic stand ing?

Do you give much or little time to class demonstrations and experiments?

Is individual laboratory work required or optional? How many hours a week are given to it? How many laboratory hours are counted as equivalent to one recitation hour?

How large laboratoly sections are handled at any one time? Do you work alone, or do you have regular paid assistants or student assistants?

Does labolatory work run throughout the yeal or is it begun after a peliod of class instruction?

Do you follow a systematic course of expeniments required of all laboratory students-one of your own, or a text-book?

Do you think it well for all students to work at the same time at the same experiments or each individually (or in small groups) at a separate problem?

Do you have the laboratory students toward the end of the year undertake anything like minor research problems or the repetition of special experiments from research literature?

Are you responsible for classes in any other subject than psychology?

Laboratory equipment: Number of rooms, large or small.

Are you pretty well equipped or do you have to make use of a good many makeshifts? Have you a workshop?

Have you a fairly adequate appropriation for running expenses or are you cramped?

\section{E. Possible improvements in the first year's work in psychology:}

In what direction would you like to see the first year's work in your own institution develop? What would you change if you had a free hand?

What do you find the chief difficulty in giving such a course as you would like?

On the instructional side-immaturity of the students, lack of preliminary training on their part in physiology, physics and other sciences, 
lack of skill in introspection, no text-book of precisely the right scope, insufficient time allowed in the programme of studies?

On the material side-inadequate equipment, poor quarters, insufficient help?

Do the students fail to take psychological experiments seriously, fail to get what the experiments mean, are shoit in manipulative skill, are unwilling to give the time necessary for careful experimentation?

\section{F. Philanthropical:}

Have you a general list of reading in periodicals and the like, outside the ordinary psychological texts, which you have found useful in the work with first year classes and which you would be willing to contribute tc a bibliogı aphy of recommended "outside reading," if one should be appended to the report of the committee?

Have you similarly any especially good demonstrations or simple experiments, not found in such good form (ol not found at all) ir the manuals, which you would be willing to publish in the report, or otherwise, for the general good?

Have you any special pie, es of demonstrational apparatus or apparatus for student practice courses which you have found especially convenient and which have not yet been described?

Finally, have you any suggestion. not extorted by the above querics as to what ought to be done in a first year's course in psychology or as to points on which this committee could obtain information that would be helpful to you?

Most of those who replied answered the questions as given, a few however took advantage of the latitude offered and gave the information in other forms. In the minds of the sub-committee such freedom of reply was more valuable than an enforced uniformity which would have allowed a statistical treatment of the data. In what follows, therefore, we shall deal with rough proportions only and not attempt tabular statements.

In presenting this report the sub-committee wishes to make grateful acknowledgements to those in charge of the work in psychology, in the thirty or more institutions responding, for the friendly coöperation without which the undertaking would have been quite impossible.

The following institutions responded: Brown, Bryn Mawı, California, Cincinnati, Chicago, Clark College, Columbia, Cornell, Harvard, Hobart, Illinois, Iowa, Johns Hopkins, Michigan, Minnesota, Mt. Holyoke, Nebraska, New York University, Ohio State University, Pennsylvania, Princeton, Smith, Teachers College (Columbia University), Texas, Toronto, Vassar, Washington (St. Louis), George Washington, Wellesley, Wisconsin, Wyoming, Yale. 
The typical beginner's course in psychology is a course in "general psychology." Experimental psychology comes later though in some of the larger institutions parallel courses are offered and the student, if so inclined, may work from the start in the laboratory. Our questions covered both kinds of work and a certain amount of information has been collected with reference to introductory laboratory courses. Our report will therefore consist of two sections, the firstand longer dealing with the introductory "general course," the second with the first work in the laboratory.

\section{THE GENERAL INTRODUCTORY COURSE.}

The first year's course in psychology is of ten a popular one in the sense that it gathers in a relatively large body of students. In many institutions some course in "philosophy" must be taken and psychology is the one selected. In about one quarter of those reporting, it is a definitely required course for all arts students and in nearly a third more it is required of students in the educational department, of pre-medical students and of those desiring certification as teachers. In more than half, however, it competes on an equal footing as a freeelective, and the size of the classes testifies to the popularity of the instructor and the subject.

The immense classes of the larger institutions 200, 300 or 400 , are often handled in a single body for lectures and in a number of smaller sections for quizzes or other reënforcements of instruction. Where the lecture method is not used the handling of the class in sections is of course imperative, and these immense classes would preclude laboratory work, even if no other reasons existed for its postponement.

The proportion of students following psychology beyond this first course varies greatly, as might be expected, but less than 40 per cent of the students continue in four-fifths of the institutions reporting, and but 25 per cent or less in twothirds of them. The fact that large classes are enrolled year after year shows that neither the subject nor the presentation of it is per se repellent. The small number going further 
shows rather that we have to do in these large classes with a student group whose major interests are in other directions and who make the course in psychology incidental to the main trend of their studies.

Psychology is an important branch of human knowledge, which has interesting bearings on every day life and vital relations to the work of certain professions, serves excellently to bring before the student certain fundamental questions of philosophy, and is for these reasons an efficient means of culture. All these functions are probably present in some measure in the minds of all instructors, but the main purpose of the course and the relative importance of its coeffects are variously conceived. In more than a quarter of even this group of institutions psychology is still looked upon as first of all the gateway to philosophy. In more than half, however, the science is presented for its own sake chiefly and the other results of its study (except perhaps the contribution to general culture, which is regarded as a natural consequence of a proper mastery of the subject) are looked upon as quite secondary. In nearly half considerable importance is attached to the presentation of a certain useful subject matter, and concreteness and applications are emphasized. In some the cultural purpose seems more directly in view, by which is meant, we infer, a somewhat less detailed and technical presentation than that used when the science is taught without ulterior considerations.

In about half the cases "psychological theory" (which, as we infer, was taken by most of those who replied to mean systematic psychology, or psychological principles presented in a coördinated way) receives chief emphasis. In about a third the chief emphasis is laid upon experimental and physiological matters; while in a few cases the relations to daily life are especially stressed. Only two correspondents speak of emphasizing the facts of the mental life, but this may be due to the way in which the question was phrased and it would perhaps be fair to count all that do not specify philosophical tendencies or psychological principles as tending in this direction. The central theme is of course the mind of 
the normal human adult and in some cases there is small departure from it. The mental life of animals and children and mental disease are referred to in a merely incidental way and for illustration. In a few cases, however, where the instructor's interest and equipment are adequate, some attention is given to borderland phenomena, child psychology and mental and nervous diseases. Topics of popular interest are of ten taken up with a view to combating superstition and popular error.

In about half the institutions reporting there is at least one instructor devoting himself to psychology exclusively. In the larger institutions with parallel courses, the instructor giving the general course gives courses also in one or more of the philosophical branches, the work in psychology being his less important function. The instructor giving the more special (experimental) course is usually free from such entanglements. In a few cases in the less differentiated departments the instructor in psychology takes a single class for his overburdened philosophical colleagues, usually in logic, ethics or æsthetics; or else carries work in pedagogy.

As regards method the course is usually a mixed text book and lecture course, though in some cases a text alone or lectures alone are mentioned. Demonstrations are frequent, especially when the central nervous system and sensation are under consideration, and in a few cases simple experiments are made in the class or by the students individually at home, e.g., such as are found in Seashore's manual. Actual laboratory work is not usually attempted. Of the other pedagogical devices, discussions (probably the ordinary class discussions are meant) are most frequently mentioned; and collateral reading, theme writing, student reports and individual conferences follow in the order named. The quiz appears in some form in nearly every report. Sometimes it is written, sometimes oral, some times it occurs once or twice a semester and is an hour long, sometimes weekly or of tener and occupies 5 or 10 minutes. In one college all the papers turned in, in a monthly test, are corrected and returned to the students, in another, where a five minute written quiz is employed, the plan calls for the 
marking of but one-quarter of the papers each time. In about a third of the institutions reporting something in the way of an outline or syllabus or special set of review questions is furnished the student as an aid. A happy variant of the review questions is that of issuing questions which cannot be answered directly from the text or lectures and which require some active response on the part of the student. In one instance this has gone even a step further and questions are given which demand a certain amount of simple introspection or experimentation or both. In this way a real acquaintance with mental facts is cultivated.

With so much in recall of the generally familiar situation, let us hear the instructors on their chief hindrances and what they would change if they had a free hand.

Inconveniently large classes or a lack of assistance is mentioned as a serious difficulty by about a third of those reporting, but this is perhaps less an evil than the dearth of students, which is once mentioned. Lack of equipment or inconvenience in quarters is mentioned by about a third, also. More than half find the students ill prepared for psychological work, especially in being unable to use the knowledge of physics and physiology which they are supposed to possess, and in heir inability to introspect. Some find themselves hindered by the mixed and uneven character of the classes in these and other respects. One or two mention the student's unwillingness to work, or his overinterest in the practical (pedagogical) aspects of the science. More, however, report no lack of enthusiasm on the part of the students, and one or two feel that immature students are the teacher's excuse for being, or state as their chief difficulties their own subjective limitations. The lack of a first rate text book is mentioned a number of times and insufficient time in the program of studies perhaps as often. Only two mention specifically the hostility of superior officers to scientific psychology.

In answer to the question as to how they would like to alter their courses if free to do so, about one-half the instructors in question reply that they would make the work more concrete and tangible and especially would add more demon- 
strational and experimental work; one or two would even like to see laboratory work required of every student as in chemistry and physics. Nearly a third would be glad of more assistants in order that the classes might be handled in smaller sections and the students receive more individual attention.

Some of these difficulties and desires are by no means peculliar to psychology, and could be matched from almost any department in any growing institution. Too large classes, too few assistants and unsatisfactory quarters are the common lot of nearly all instructors in nearly all sciences. The same is true in a measure of the immaturity and imperfect training of the students. The suggestion, however, that their deficiency in these particulars is only a sign that they need a teacher meets the matter in part only: there seems to be a real difficulty here and one resting with especial weight on psychology. Inability to introspect means inability to get at the subject matter of psychological science and points to an unusual danger of substituting a knowledge of words and descriptions of psychical phenomena for knowledge of the things themselves. This in the committee's opinion is a matter of first class importance and needs the attention of every teacher of beginners in psychology.

The frequent desire for more demonstrations and more experiments in the beginner's course may possibly mean a wish for a more striking lecture material only, but it ought to mean a desire for a closer envisagement of the psychic facts by the pupil.

The fact that many students whose main interest lies elsewhere are now drawn in to the first year's classes in psychology and that introspection is a hard matter at the best would seem to make the opening of their inner eyes to the mental world one of the first and most imperative duties of the course. Practical bars of the most insuperable sort hinder the accomplishment of this by regular work in the laboratory. The classes are almost everywhere too large and the amount of time which the students can give to psychology too small. What is wanted is clearly some other method of bringing the student into contact with the psychical facts. Something is 
already being done, and of ten a good deal, in the way of demonstrations and class experiments and this is in every way admirable when the instructor makes use of the introspective possibilities which they offer and drives home the psychological meaning of what is presented. But the beginnings which have been made in the issuing of questions requiring simple introspections and experiments, and in the preparation of simple experimental courses which can be followed by the student at home as a part of the regular preparation for the lecture or recitation hour, are undoubtedly still more important steps in the right direction and worthy of all encouragements.

The most interesting and valuable of the information brought out by our questions was that which a number of our colleagues were kind enough to give in reply to a portion of our last question which read as follows:

"Finally, have you any suggestions not extorted by the above queries as to what ought to be done in a first year's course in psychology?" Several of these paragraphs of suggestion we desire to give in extenso.

SUGGESTIONS FOR THE IMPROVEMENT OF THE GENERAI COURSE.

I. "But several things are needed for the improvement of courses like A (a "general course"). . . . I might mention:

"( ( ) A good reliable list (a) of inexpensive demonstrational apparatus and (b) of useful demonstrational experiments, whose use with the class would occupy a very small prcportion of its time, but would serve not merely for its entertainment but as a real help in comprehending the facts and principles discussed. I am not fully satisfied yet with existing lists.

"(2) A good critical bibliography on each phase of the subject.

"(3) A standard set of lantern slides.

"(4) A larger agreement among the leading psychologists as to what are the fixed and permanent underlying principles of the science.

"(5) An adequate text book that will emphasize these fundamental principles first of all, aiming to present them soundly and convincingly with only enough of detailed fact to make these essential principles clear; leaving to lectures and collateral reading the acquisition of further details and the application of the fundamental principles to them. At present to reach this ideal, I suppose each of us must write his own text book; for I haven't found one yet that satisfies me."

II. "I am of the opinion that a General Introductory Course that is required should not be a laboratory course. Too little can be covered in such a course and to my mind the advantage of the laboratory method should, under the circumstances, be sacrificed for the sake of the greater 
advantages of a course of wider scope, and such as can be given in the same time in lectures supplemented by copious demonstrations.

"If the course is not required and if most of the students take further work in Psychology the force of the above objection is gieatly reduced. Nevertheless, even in this case, a general lecture course with demonstrations and class experiments is to be preferred by way of introduction to a strict laboratory one.

"I insist in my general required course upon the structure, the organization and the function of the nervous system. This seems to me requisite not only for a proper understanding of psychology as a science, but also for a modern understanding of man and his activities. This general culture value of a clear and not too scrappy understanding of the organization and of the function of the nervous system is, to my mind, of the first importance in a required course."

III. "I. Business-like administration is desirable in the interest of the student, who is likely to get into slovenly habits. Tc this end $\mathrm{W}$ - has found nothing better up to date, than dividing each hall year's work into 5 parts, each part being a subject, such as space perception, association, memory, reaction time; the work on each part is to be completed at a previously set date, the laboratory notes to be handed in, and an examination on that part taken.

"2. Instead of assigned cultural readings W-- prefers, usually, problems to be worked out by the student; as for example (a)illustrations of Weber's law from common life (usually not very successful); (b) records of trains of association; (c) exercises in finding average, constant and variable errors, etc."

IV. "Standardize by getting (and recommending) good sense organ and central nerrous system slides and models. By suggesting certain sets of demonstrations. . . . (but) leaving time for instructorial bent.

"Arrange some exchanges of first year examination papers between different univer sities."

V. "I would suggest that the committee prepare or further the preparation of leaflets or pamphlets containing directions for single experiments or groups of experiments in order that instructors may make any combination suited to their needs. Even the smaller manuals contain much not suited to some classes."

VI. "I would suggest that a loose leaf laboratory book be prepared, corresponding to books now used in physics, chemistry, etc., describing a series of simple experiments and containing all the material necessary for performing the experiments. If the experiments were performed under constanl conditions, and the records sent to some central body, we should be in possession of some psychologic norms with which we could compare the results given by our own students. I have a lot of this stuff worked out now and should be glad to coöperate."

VII. "The thing that more than any other keeps us to the old lines is the belief that the beginning student should get a firm grip on the vocabulary of psychology, even if that vocabulary is somewhat archaic, and that a study at first limited to the normal, human, adult mind, is best for that purpose." 
VIII. "If my observations are correct Experimental Psychology is now taken (elected) by only a few students. The way to save this work for education is to combine its essentials with the introductory course, I sincerely believe. Experimental psychology has an 'educative' value in various ways but now this is almost entirely lost. Combining the essentials with the introductory course would also assist in giving the essentials of the text book. I used Seashore's Elementary Experiments in my beginning classs this year and found it decidedly helpful."

IX. "With respect to ordinary college fir st year psychology my main divergences from customary opinion are:

"(I) That this course in psychology is not to make philosophers.

"(2) That this course in psychology is not to make psychologists.

"(3) That this course in psychology is to make men and women better fitted to understand all the sciences and arts dealing with human nature.

"(4) That psychology is not best taught to beginners as the study of human consciousness but as the study of human nature and behavior.

"(5) That experiment dealing with realities and exercises testing knowledge and power by demanding their application to new problems are a sine qua non of success in teaching psychology (or anything else).

"(6) That the function of the teacher is not primarily to get a great amount of work done by the students but to get the greatest amount of knowledge, skill, etc., from a given amount of work by them.

"(7) That the actual content of the course is more important than most teachers of psychology think and the form or discipline or point of view, etc., less important."

$X$. "If conditions permitted it, I should be in favor of giving a whole year to the required course, in which the only labolatory work would be of a practice character. In such a care I would like to see the dynamic, rather than the static, aspects of mind emphasized. I would begin the course, not with a detailed analysis of sensation but with a consideration of the biological functions and place of the human mind. Then I would proceed to more careful analysis with experimental illustration. I would spend a good deal of time on the psychology of conduct, thinking, feeling, bringing out the connection between ethics, logic, æsthetics, etc., and psychological analysis. I would give some attention to abnormalities of consciousness and action and point out the practical, social, moral and educational bearing of psychology.

"Whatever may be said in favor of a structural psychology that is rigorously atomistic, as a legitimate scientific proceeding. . . . my experience as a teacher leads me to say that emphasis on the stiuctural, static, and atomistic points of view is not desirable in a first year course.

"Whatever the value or interest of these very vague remarks, they are to be taken as the expression of a teachel who, while 1ecognizing the independent rights of psychology, does not deem it wise, from the standpoint of undergraduate instruction and of philosophical culture, to divorce if from philosophy. In fact, as one whose interests are predominently epistemological, ethical and metaphysical, I am not at present able to draw any sharp dividing line between functional psychology and philosophy." 
XI. "A satisfactory consensus of opinion, today, I think is impossible to obtain; and such as you will get I consider more liliely to do harm than good, except possibly in the smaller colleges. . . . I consider the attempt to standardize courses premature; let the men in the field expeniment with courses, the best will survive; but your made to ordet course or your average cou-se will never be as gond as the best."

XII. "I do not think that it is possible to teach a science in any other way than as a "science to be cultivated for itself." That is to say, I should give precisely the same introductory course, whatever the aims and needs of the students who took it. I doubt whether it is advisable to cut a science to suit the requirements even of a distinct professional course; I doubt, that is, if it is advisable to teach English Literature for Engineers or Physiology for Medical Students; I believe that the best results are gained, in such cases, by teaching English literature and physiology. But 'general culture' or 'liberal education'-the supposed main object of college courses in arts and sciences--is not a profession, is not at all strictly definable; and I do not see how it is possible, not to say desirable, to cut psychology to fit that requirement.

"I teach psychology, at the beginning, in an elementary manner. I make things as simple as possible; I omit phases of problems, even whole problems, where simplification to the necessary degree is impossible; I am dogmatic on points where dogmatism is strictly out of place, although the dogmatic statements are always qualified for those who have ears to hear. But I am all the while teaching psychology, as best I can, without ulterior motive or ideal. I get a good many men from engineering, medicine and law; the number of these outside students is steadily increasing; but I make no concessions to them.

"If any other ideal is followed, it seems to me that one of two things must happen. Either the teacher lapses into dilettantism; and for this there is no defence. Or the teacher substitutes his personal and private judgment for the objective judgment embodied in the actual course and growth of the science; he forces on the students his own notion of cultural or philosophical or practical application, instead of showing what has been accomplished and allowing that accomplishment to speak for itself. In general, this alternative must lead to bad results.

"I grant, of course, that a teacher, a man who is in love with his subject, will do well with students whatever method he follows and whatever choice he make. That is axiomatic. But I suppose that the aim of this questionary is to help the weaker brethren, and not to legislate for those who already know. And I should accordingly counsel the weaker brother, if he is a professor of psychology, to teach psychology. If he does this, up to his honest limit of achievement, he will find that psychology will show its own bearing upon culture, its own philosophical relevance, its own applicability. These references may then be followed up, as needs arise and as the size of the staff allows, by later courses, given either by the department of psychology or by those of philosophy and education, etc. To interject them at the beginning is to warp the mind of the student.

" $M y$ ideal is the local separation of the college from the university. 
With this ideal realized, I should offer (I) an introductory course, much as I do now, for graduates in other departments, and (2) research and training courses in the laboratory. I should not, by choice, be a 'college' professor. Being this, however, I have rigorously pushed my university ideals into the college work. The success is patent; my 270 elective students-if their number is compared with the number of the sophomore students in the college-are sufficient witness. To say that hard work cannot be got out of the students, or to say that scientific psychology is uninteresting, is simply to cover up one's own laziness or incompetence.

"I must here interject my ideas on the lecture system. The lecture has a twofold advantage over the recitation. (I) It is economical, since one man handles a large number of students; the method of recitation is extravagant. This fact alone will mean the retention of the lecture system, wherever it can possibly be employed with success. (2) It is educationally the better method, for the average student and the average teacher. For the reconstruction of a lectule from notes means an essay in original work, in original thinking; while the recitation lapses all too readily into text-book rote and verbal repetition.

"It is, nevertheless, true that sophomore students are on the whole inadequate to a lecture course. They cannot take notes; they cannot tear the heart out of a lecture. (They are also, I may add, inadequate to the reading of textbooks or general literature, in much the same way.) Hence one has to supplement the lecture by syllabi, by lists of questions (indexes, so to speak, to the lectures), and by personal interviews. I spend, on the average, $S$ hours of time on every one of my sophomore lectures; I give 24 hours a week to a 3 houls' course. But, doing this, I secure a fail-percentage of 2 or under.

"Evidently, this method is wasteful of good material. While it costs less than it would cost to add half a dozen assistants for recitations; and while it is also, educationally, a better method than that, it still wastes good professolial time. The remedy, is, to accustom students to notetaking in the high school. In the English public schools, a part of the work in the upper forms is lecture-work, for which the boys are held responsible; they therefore go to the univer sity in some measure prepared for the lecture courses. Now lectur es are frequently given, by outsiders, to the students in American high schools; but they are looked upon as a recreation or a bore. I suggest that these lectures, given once or twice a term through the four years, might be utilized for teaching the students to take notes. The students might, in a minor way, be held responsible for the reproduction of theil contents. Then, in the freshman year, the student might take, say a single lecture course of 3 hours a week; so that, in the sophomore yeal, he would come to the psychologist with some training.

"I speak of averages all the way through. A man may be a born lecturer, or a born user of the Socratic method; he will succeed anywhere. But we do not get congenital ability throughout our faculties. I believe in the lecture system, for the average student and the average instructor of our subject, but I suggest that the present state of affairs might be im- 
proved, on the side of the student, by better high school pieparation. As for the instructor, he must be taught, as sharply as necessary, that a lecture is a work of art, and not a perfunctory delivery of information. Murh of the failure to interest, in psychology, is due to the instructor's lack of education in his art: he cannot manage his voice, he has not learned what he wants to say, he does not properly enunciate, he cannot vary from narrative to exposition, to argument; his anecdotes are purple patches and not relevant lightenings of the material. Time and again I have listened to 'lectures in psychology' that made me wish I had the lecturer alone, with nothing but the moral law in my heart and the universe of stars above me.

"So-to return-my chiet difficulty is immaturity of training (not of mind) on the part of the students. Physiology and physics they have tasted, or can get concomitantly; introspection it is my business to teach them; text-books I can write myself; and I have my share of theil time. I want them only to know how to deal with spoken or printed material.

"Our equipment I have already described. Students who lack seriousness, or understanding, or manipulative skill, or time to devote to the subject, are bowed out of the laboratory in the course of the first fortnight. Why should we bother with them? They are not obliged to come in, and we are assuredly not obliged to hold them if they do come in. We take all imaginable pains with the real student, whatever he may at first be lacking in; but the unfit are eliminated. And if one has had experience, the spotting of the unfit is not a very difficult thing.

"I should deprecate the publication of any list of first year's collateral reading. The science is growing by leaps and bounds, and the collateral reading shifts and changes from year to year. To publish a list would be to relieve the instructor, for some years to come, of what should be an integral and an interesting part of his own work and growth. The list would remain, fossilized, and students would suffer. No concessions should be made, I think, to the weakness of the instructor; every allowance should be made for the poor training of the student. . . .

"The sum and substance of my recommendations is that you provide a competently trained instructor, and let him teach psychology as he best can. What the student needs is the effect of an individuality, a personality; and the lecture system provides admirably for such effect. I should strongly deprecate the issuance of any general plan of organization, or the authoritative 1ecommendation of any special topics or procedure, which should tend to mechanise instruction in the colleges. We are overorganized, ovel-businesslike already. I venture to suggest that a great danger in the Report of the Committee lies precisely in this point. If its recommendations are over-stringent, if the fieest play is not allowed to the instructor's personal training and individual capacity, we shall be out of the frying pan into the fire."'

1 Several correspondents would like to have information as to particular questions with reference to the first year's course-as, for example, the preferred order of presenation of the various topics in the course, whether introductory experimental work hould begin with bare sense-experiments, how far psychologists feel an unsatisfactory 
Recommendations. The Committee has no such idea of its function as that implied in the warning of our colleagues who penned extracts XI and XII above. It does not desire to be responsible for an average course or to standardize the courses now given. It agrees unreservedly with the writer of extract XII that the best way in which to secure good courses in psychology is to select well trained men and give them a free hand. Good teaching is an art and the teacher must be assured the artist's freedom. The Committee's function, as we conceive it, is not to establish norms of any sort, but to formulate more clearly certain ideals on which many or all of those teaching psychology are already agreed, to point out means by which these can in some measure be realized, and finally to recommend to the Association such action as will assist those who care to work in the direction suggested.

The ideal which the Committee desires to formulate and for which it hopes the endorsement of the Association is that of the fullest possible acquaintance on the part of the student with the concrete facts of consciousness as the rational basis for a knowledge of psychology-an ideal which has been frequently mentioned by our correspondents and which is shared we believe by all or almost all of our colleagues. As means to this end the Committee urges, as indicated above, the fullest use of demonstrations and class experiments, given always in such a way that the student shall not fail to grasp their psychological meaning, and the development of the scheme of simple home experiments and introspections already in tentative use by at least two of our colleagues.

In furtherance of these ends it recommends to the Association the formation of two permanent committees, one on Demonstrations and Class Experiments and another on Psychological Experiments Outside the Laboratory. The functions of the first should be to publish from time to time in some one of the psychological journals, first a classified bibliography of such good demonstrations and class experiments

tendency toward practicality in the students which come to them from pedagogy, and how far they still suffer from a tendency of philosophers to dominate the departments to which the psychological courses belong. 
as already exist in readily accessible literature, second to collect and publish in full in the same way directions for making such demonstrations and class experiments as are found only in relatively inaccessible literature, and third to collect and publish in the same way similar demonstrations, experiments and descriptions of demonstrational apparatus as are now in use in American laboratories and as yet unpublished. (Several of our correspondents have already signified their willingness to contribute to such a collection. ${ }^{1}$ ) The Committee on Experiments Outside the Laboratory should undertake the same functions with reference to their particular topic. Our purpose in suggesting that these committees be made permanent is that they may be given time to work slowly at the material to be gathered and publish from time to time as it is collected without producing at once, or waiting for, anything approaching a complete exploitation of the field; and that the experiments and demonstrations which they publish may be kept up to date.

\section{THE FIRST YEAR IN THE LABORATORY.}

In the half dozen or more institutions in which parallel introductory courses are offered, it is possible for the student to begin the subject of psychology with its experimental aspect. But even in these cases it is exceptional that a student enters the laboratory before he has taken the general introductory course, though the two courses are sometimes carried at the same time. In all other institutions where laboratory work is given at all, it follows an introductory course. The first laboratory course usually runs through a full year though in some instances it is given in a single semester.

The preferred size of laboratory sections is ten or under, though in some institutions, where the work has been thoroughly systematized larger sections (up to 30 ) are handled by the instructor with one or more assistants. Practical

\footnotetext{
${ }^{1}$ We would suggest for membership on this latter committee our colleagues Professor J. E. Lough of New York University and Professor Margaret Floy Washburn of Vassar College who have already used with success the methods in question.
} 
reasons, especially the cost of much duplicating of apparatus, make it necessary in most cases where the laboratory sections are at all numerous, for different groups to work at different problems, but some instructors in beginning the laboratory course have all the students work at the same problem at the same time until several problems have been covered; and in exceptional circumstances it has been found best to carry this method through the major part or even the whole of the beginner's course.

After a year or so of laboratory practice several teachers set students at repeating experiments from research literature or assign them minor research problems under supervision. It is rare, however, that this can be done within the first year.

In the institutions in question the number of rooms used for laboratory purposes ranges from $\mathrm{I}$ to 32 , but in the latter case of course many rooms are included which are not used for the first year's laboratory courses. The most frequent number mentioned is 5 , and half of all reporting give numbers from 2 to 7 . In two institutions the laboratory consists of one room only though in one of these cases other rooms may be used in time of need.

In equipment most of the laboratories supplying information (about two dozen) are at least fairly equipped for the sort of work required. Only three speak of their equipment as inadequate, and one of these is just starting.

Appropriations are felt to be inadequate and cramping in but four of twenty eight; and some small laboratories are able, by economy and by concentrating research upon a single topic at a time, to do work of excellent quality, both practice work and research, with the expenditure of relatively small sums.

With reference to the constitution of the first year's laboratory course there is a wide spread diversity. Eclectic courses are common and it is once or twice remarked that the course is varied from year to year or should be adaptable to the needs of the students taking it. It must of ten be limited also by the equipment, or even the physical situation, of the laboratory. 
The data collected with reference to the first year's work in the laboratory, while it shows that most of the laboratories reporting are in a fairly prosperous condition is sufficient to to justify the Committee in but a single recommendation.

Recommendation. In view of the diversity of interest, requirement and possibility the Committee looks with favor upon the suggestion of one of its correspondents that a laboratory course should be prepared on the principle of the "loose leaf" courses now used for elementary laboratory work in other sciences, by means of which an instructor can vary his course with ease and within wide limits. The preparation of such a course is not the work of the Association nor of a committee working under its authority, but we suggest that, if so moved, the Association might further such an undertaking by expressing its official approval of the plan. 